\title{
Specification of direction and extent in motor programming
}

\author{
MICHEL BONNET, JEAN REQUIN, and GEORGE E. STELMACH \\ Department of Experimental Psychobiology, Institute of Neurophysiology and Psychophysiology \\ National Center for Scientific Research, Marseilles, France
}

\begin{abstract}
A partial advanced information paradigm that utilizes movement precues about upcoming movements was applied to flexion and extension foot movements of either a large or small extent. By analyzing reaction times obtained when precues gave either no information, partial information about either direction or extent, or complete information about movement parammeters, it was found that initiation times varied as a function of amount of advanced information. Reaction times were fastest in the complete information condition, followed by the partial and no information conditions. With regard to type of parameter to be programmed, extent was faster than direction. Further, the data suggested that direction specification and extent specification must be considered as two serially organized, interdependent, and ordered operations, with the former consuming more time than the latter.
\end{abstract}

It is commonly viewed that the role of a motor program is to prescribe values on certain kinematic parameters that are under program control. In examining this prescription process, Rosenbaum (1980) extended the longstanding partial advanced information paradigm (Leonard, 1958) to examine the operational characteristics of motor programming. The major question addressed was how movement dimensions, such as arm, direction, and extent, are specified. Using a precuing technique, subjects were required to execute simple limb movements that varied in a number of movement dimensions (e.g., arm, direction, and extent). The precues signaled total, partial, or no information about the intended movement, and by comparing the possible combinations of precues, inferences were made about the independent and interactive roles of each movement parameter in motor program construction. From his data, Rosenbaum concluded that specifications of the various movement dimensions are serially organized operations, but without a fixed order, and that programming times are longer for arm than for direction and longer for direction than for extent.

Do these results really address response programming construction? Recently, Goodman and Kelso (1980) and Stelmach and Larish (Note 1) have criticized the technique used by Rosenbaum because it possesses a substantial cognitive-motor translation process. Rosenbaum used letters to precue the subject, and previously learned color-coded labels were signals to respond, raising the possibility that translation (verbal code to position code) processes (Teichner \& Krebs, 1974) may have influenced the produced reaction times. In instances in which the reaction stimulus and its associated response are indirect and relatively unpracticed, nonmotor, cognitive pro-

Reprint requests should be addressed to Michel Bonnet, CNRS-INP 3, B.P. 71, 13277 Marseille Cedex 9, France. cesses (stimulus-response translations) make an unavoidable and significant contribution to reaction time. To overcome this potential problem, Goodman and Kelso (1980) and Stelmach and Larish (Note 1) visually precued the subjects directly with compatible stimulusresponse mappings, thus minimizing the translation processes required to execute a response. Both of these studies found that although the reaction times decreased as a function of the number of parameters precued, there were no systematic effects of precuing on direction and extent parameters.

In the experiment reported here, precues were presented such that there were compatible stimulusresponse mappings in a reaction time situation in which flexion and extension foot movements were employed. After the partial advanced information was presented, the subject had to program nothing, the direction or extent of the upcoming foot movement, or both the direction and extent of the upcoming foot movement. The primary issue addressed concerned whether the specification times for movement parameters of the foot would be different and whether different specification times would be found within a movement parameter.

\section{METHOD}

Five right-footed subjects participated in the experiment. Each volunteered and was not paid for services.

The experiment took place in a soundproof experimental chamber with the subject sitting in a semireclining position in an armchair. One meter in front of the subject at eye level were four light-emitting diodes, each $3 \mathrm{~mm}$ in diameter, mounted vertically beside the circular display screen of an oscilloscope. The arrangement was such that the CRT beam remained visible, except when it moved behind one of the diodes that were mounted 2 and $4 \mathrm{~cm}$ above or below the center of the screen. The subject's right foot was attached to a moving pedal that rotated against a spring, around the axis of the ankle. This pedal rotation controlled, through a potentiometer, the vertical displacement of the CRT beam, at a rate of $2.5 \mathrm{deg} / \mathrm{cm}$. When the 
subject's foot was at rest, the potentiometer was adjusted in order to locate the beam in the center of the screen. Dorsal flexion and an extension of the foot resulted in an upward and a downward displacement of the beam, respectively, toward the light-emitting diodes.

The subject's task was to dorsal flex or extend the foot as quickly as possible when the imperative stimulus appeared. Movement initiation times were recorded by a light meter activated by the displacement of the moving pedal. Since subjects were instructed to move as fast as possible, movement accuracy was continuously monitored. However, it was not precisely measured.

Two seconds before the response signal, a warning light was presented for $1 \mathrm{sec}$ and was generated by the lighting of one, two, or four diodes in order to provide the subject advanced information about the movement to be performed. The four programming conditions used were (1) Program None, in which both parameters were known prior to response initiation (this occurred when the warning signal specified the exact movement to be performed), (2) Program Extent, in which direction was known and extent remained unspecified prior to initiation (this was signaled by lighting both diodes above or below the center line), (3) Program Direction, in which extent was known and direction remained unspecified prior to initiation (this was signaled by lighting either the two short or the two long targets), and (4) Program Direction and Extent, in which no prior information was given (this was signaled by lighting all the warning lights at once).

The experimental design required each subject to perform the task for five successive daily sessions. Each session was formed by four successive series of trials separated by a 10-min rest, and each series consisted of 80 trials. Since the task became a simple reaction time condition when the warning signal gave full information about the movement to be performed, the response signal did not occur in 16 "catch" trials, in order to prevent anticipatory reactions. In the 64 remaining trials, the four possible response signals were equiprobably and randomly presented in order to counterbalance sequential effects up to the third order. Each type of response signal was equiprobably preceded (i.e., four times) by a warning signal giving no information, partial information about movement direction, partial information about movement extent, or full information. Within these constraints, presentation order of the different warning signals was randomized as far as possible.

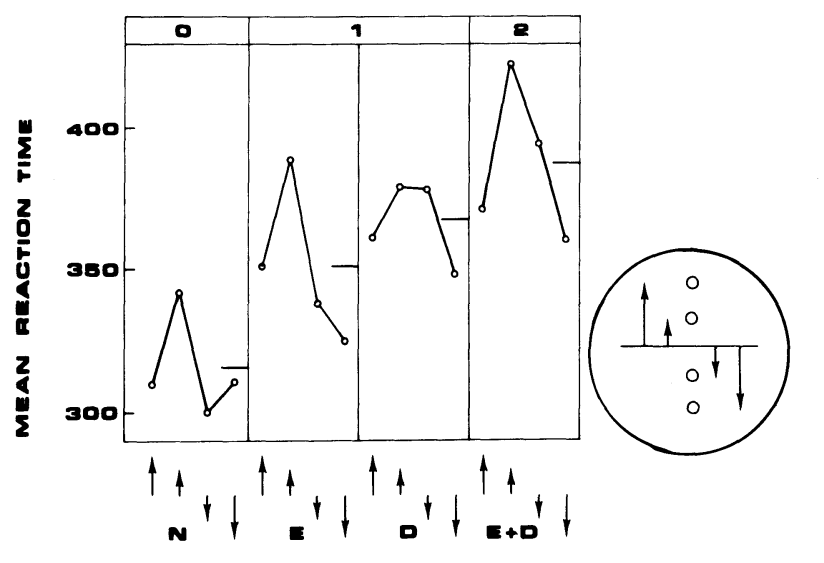

VALUES TO DE DPECIFIED

Figure 1. Mean reaction times plotted as a function of the values to be specified. On the right, there is a graphic display of how the precues were presented.
Table 1

\begin{tabular}{lccccc}
\hline & N/E+D & E/D & D/E & F/N & Mean \\
\hline Short & 408 & 379 & 363 & 321 & 368 \\
Long & 366 & 354 & 338 & 310 & 342 \\
Mean & 387 & 367 & 351 & 316 & \\
Flexion & 397 & 370 & 370 & 326 & 366 \\
Extension & 378 & 363 & 332 & 306 & 345 \\
\hline
\end{tabular}

Note-Column headings are expressed as advance information/ values to be specified: $N=$ none, $E=$ extent, $D=$ direction, and $F=$ full.

\section{RESULTS}

The mean reaction times for the four programming conditions are plotted as a function of parameters to be specified in Figure 1. Figure 1 also displays the breakdown of response movement parameters (direction: dorsal flexion and extension; extent: short and long) across all precue conditions. As can be seen in the graph, there is a large main effect of precue $[F(3,12)=35.69$, $\mathrm{p}<.001]$. The mean reaction times for the advance information conditions were Program None, $315 \mathrm{msec}$, Program Extent, $351 \mathrm{msec}$, Program Direction, $367 \mathrm{msec}$ (with a combined mean of $359 \mathrm{msec}$ ), and Program Direction and Extent, $387 \mathrm{msec}$. These results are in general agreement with those of Goodman and Kelso (1980), Rosenbaum (1980), and Stelmach and Larish (Note 1). That is, as the number of stimulus-response alternatives was reduced, there was a corresponding reduction in reaction time.

When the direction of the movement actually performed is considered, reaction time was shorter for extension $(345 \mathrm{msec})$ than for flexion $(366 \mathrm{msec})$ movements $[F(1,4)=11.19, p<.05]$, and this difference depended on the parameters to be specified. As is apparent in Table 1, when direction had to be specified, the difference was minimal $(7 \mathrm{msec})$, and when extent had to be specified, the difference was substantial $(38 \mathrm{msec})$, generating a clear interaction $[\mathrm{F}(3,12)=7.90, \mathrm{p}<.001]$. Additionally, when the extent of the movement actually performed is considered, reaction time was shorter for long movements $(342 \mathrm{msec})$ than for short movements $(368 \mathrm{msec})[\mathrm{F}(1,4)=18.24, \mathrm{p}<.05]$.

When looking within the single level of parameterization for direction and extent, it is found that direction took longer to be programmed than extent. This difference was $16 \mathrm{msec}$ and was significant $[F(1,3)=9.85$, $\mathrm{p}<.05]$. However, this difference occurred only in the extension movements; careful examination of the data will reveal that there were no direction and extent differences for the flexion movements and that the entire effect $(31 \mathrm{msec})$ was located in the extension movements. Further, when this difference was isolated in the long and short movements, it was clearly present for both types of movements, as each mean difference was $16 \mathrm{msec}$. Consequently, the interaction between the effects of partial information (direction vs. extent) and 
direction of the movement actually performed (short vs. long) was statistically significant $[\mathrm{F}(1,4)=22.91$, $\mathrm{p}<.01]$.

\section{DISCUSSION}

Rosenbaum (1980), using a partial advanced information paradigm, recently set out to understand movement selection processes by examining the temporal components of their operation. He raised such questions as: Are the values defining a movement specified serially or in parallel? If the specification process is serial, are the values specified in a fixed or variable order? Are the times to specify different values on a given control dimension the same or different? While Rosenbaum raised other interesting issues, we felt the foregoing were the most pertinent to our data. The data collected in the present experiment allow some observations about motor programming construction with regard to movement parameterization: the independence of the specifications, the seriality of the operations, and the order in which these parameters are specified.

With regard to specification times, it should be pointed out that when subjects had complete information so that nothing had to be programmed, there were large differences between short and long movements, as well as extension and flexion movements. These differences cannot be related to differences in specification times only. More likely, the observed differences largely depend upon characteristics of movements themselves and upon their underlying muscular activation process. The movement-extent differences found are probably due to the functional properties of the agonistic-antagonistic muscular apparatus of the leg. It is rather difficult to make very short accurate movements with the foot. Thus it is likely that the short movement extents were so small in terms of angular rotation that they probably did not start with a ballistic phase, whereas the long ones did have a ballistic phase. Moreover, the primary antigravity function of the soleus and gastrocnemius muscles compared with the tibialis anterior probably makes foot extension easier than foot flexion.

The difference between reaction times observed when either direction or extent was precued alone means that specification time for direction is larger than specification time for extent. While these results agree with Rosenbaum's (1980) findings, they disagree with those of Goodman and Kelso (1980) and Stelmach and Larish (Note 1). The reaction time difference observed in the present experiment was $16 \mathrm{msec}$ smaller than the 25 -msec differences observed by Rosenbaum. It is considerably larger than those found in the Goodman and Kelso and Stelmach and Larish papers. Further, it should be noted that the study presented here is the first to report differences in specification times for direction and extent in spatial-compatible mapping situations. We believe that, due to the nature of the foot apparatus and its biochemical properties, it takes considerably longer to specify a movement direction than to specify an extent of movement.

The data indicate an interaction between the parameters to be specified. The increase of the difference between reaction times for short and long movements from the condition in which both parameters were known $(11 \mathrm{msec})$ to the condition in which extent remained to be specified $(25 \mathrm{msec})$ means that parameterization for short extents took longer than that for long extents. Further, the fact that this difference increased when both parameters had to be specified $(42 \mathrm{msec})$ suggests that parameterizing extent was altered when direction also had to be specified. Similarly, the reduction, even the elimination, of the difference between reaction times for flexion and extension movements from the condition in which both parameters were specified $(20 \mathrm{msec})$ to the condition in which direction remained to be specified $(7 \mathrm{msec})$ means that the specification times for flexion and extension were approximately the same. Further, the increase of this difference when extent had also to be specified
$(19 \mathrm{msec})$ means that parameterizing direction is altered when extent must also be specified. Taken together, these facts suggest that specification of direction and specification of extent are interdependent.

The fact that the time for specifying direction and extent together is greater than the sum of times for specifying direction alone and extent alone confirmed the necessary condition for serial processing stages. The benefit of advanced information when the precues are considered separately is $56 \mathrm{msec}$ ( $36 \mathrm{msec}$ for direction and $20 \mathrm{msec}$ for extent), and this is smaller than when both parameters (direction plus extent) are specified in advance: $71 \mathrm{msec}$. Of course, this is not a sufficient condition for seriality because differences can be explained by the increase, from two to four, of stimulus-response alternatives. However, the fact that differences between values within each dimension when both dimensions had to be specified were generally larger than the corresponding differences when each dimension had to be specified alone makes a supplementary argument for serial processing.

Finally, since the shortening of reaction time when direction was precued appeared without the precuing of extent at the same time, and reciprocally, there is no clear evidence for an irreversible order in movement dimension specification. However, a supplementary suggestion about specification order can be drawn by comparing reaction times for short and long movements on one hand and flexion and extension movements on the other, from the condition in which both parameters had to be specified to one in which either had to be specified. The reaction time difference for short and long movements when they had to be specified $(42 \mathrm{msec})$ decreased equally when each parameter remained to be specified alone (to $25 \mathrm{msec}$ for direction and extent). On the contrary, the difference in reaction time for flexion and extension movements from when both parameters had to be specified $(19 \mathrm{msec})$ was suppressed when direction remained to be specified $(7 \mathrm{msec})$, whereas it increased when extent parameterization remained to be completed $(38 \mathrm{msec})$. Therefore, the time required to specify extent varied according to movement direction, whereas the parameterization of direction did not vary as a function of direction. This suggests a preferential order of specification for direction and extent, because parameterization of direction allowed differential use of extent information, but the reverse was not true for extent. This inference agrees with the often proposed view of a hierarchy between direction and extent programming processes.

Assumed in the precuing methodology is that the reaction time to make a response reflects the time to specify the movement parameters that could not be prepared in advance because the advanced information did not allow complete programming. Thus the response selection stage is thought to be the major stage of processing that is being tapped. As mentioned previously, Rosenbaum's (1980) experiment inadvertently stressed premotor cognitive processes because he used a methodology that required different stimulus identifications and stimulus-response translations. These troublesome methodological issues raised questions of whether the response selection stage was responsible for his precue findings. Since the present study found differences in the specification times between direction and extent, when there were few differences in stimulus identifications and stimulus-response mappings, attention may be focused on the response selection stage. Thus, despite some troublesome problems, it is felt that, if used properly, the precuing methodology can be useful in assessing specification times for movement parameters. However, as in other experimental situations, there are other ways to assess specification times for response programming (Larish \& Stelmach, Note 2).

\section{REFERENCE NOTES}

1. Stelmach, G. E., \& Larish, D. D. On the relationship between response organization processes and response programming (AFSOR Tech. Rep. 3). University of Wisconsin, Madison, 1981. 
2. Larish, D. D., \& Stelmach, G. E. Preprogramming, programming, and reprogramming of aimed hand movements as a function of age. Manuscript in preparation, 1981.

\section{REFERENCES}

Goodman, D., \& Kelso, J. A. S. Are movements prepared in parts? Not under compatible (naturalized) conditions. Journal of Experimental Psychology, 1980, 109, 475-495.
LeonARD, J. A. Partial advance information in a choice reaction time task. General Psychology, 1958, 49, 89-96.

Rosenbaum, D. A. Human movement initiation: Specification of arm, direction and extent. Journal of Experimental Psychology: General, 1980, 109, 444-474.

Teichner, W. H., \& Krebs, M. J. Laws of visual choice reaction time. Psychological Review, 1974, 81, 75-98.

(Received for publication October 16, 1981.) 